\title{
Plasmapheresis in Tuberculosis Treatment
}

\author{
Voinov VA ${ }^{1 *}$, Tityukhina $\mathrm{MV}^{\mathbf{2}}$, Karchevsky $\mathrm{KS}^{\mathbf{1}}$ and Isaulov OV ${ }^{\mathbf{1}}$ \\ ${ }^{1}$ I.P. Pavlov First Saint-Petersburg State Medical University, Saint-Petersburg, Russia \\ ${ }^{2}$ Moscow Center for Tuberculosis Control, Moscow, Russia \\ *Corresponding Author: Valerii A Voinov, Head of Therapeutic Apheresis Unit, I.P. Pavlov First Saint-Petersburg State Medical University, \\ Saint-Petersburg, Russia.
}

Received: June 01, 2019; Published: July 11, 2019

DOI: $10.31080 /$ ASMI.2019.02.0306

\begin{abstract}
Tuberculosis remains a fairly common infection, especially in developing countries. Despite all the successes of modern medicine, its treatment remains a difficult problem. The main reason is the increasing number of microbacteria strains resistant to most drugs. A large number of them, taken simultaneously, develop severe intoxication, which is the leading cause of deaths. Apheresis therapy can help solve this problem, which, in addition to detoxification promotes immunocorrection, too. Plasmapheresis experience shows a faster recovery of organ dysfunction and achieving a more stable remission of the disease.
\end{abstract}

Keywords: Tuberculosis; Drug Resistance; Intoxication; Plasmapheresis

\section{Introduction}

Tuberculosis is still a fairly common infection, ranging from 6 to 24 cases per 100,000 of population in the world, reaching even the level of 232-453 per 100,000 in some African countries [1-3]. And in the developed countries the observed tuberculosis incidence decline is slowing down due to migration processes become more intensive [4].

Drug therapy

Despite all the advances of medicine, tuberculosis is the ninth leading cause of death, reaching 150 million deaths worldwide with mortality rate of up to $15 \%$, although most of these deaths occur in the developing countries [5]. In most cases, death is a result of increasing multi-drug resistance of Mycobacterium tuberculosis (MT) to $\beta$-lactams and other antibiotics [6-8]. About $20 \%$ of MT is resistant to major anti-TB drugs, including isoniazid and rifampicin [9]. The increasing spread of multi-drug resistant cultures of MT is the most important issue, which is difficult to be resolved to control this infection. In such cases, the disease becomes incurable, using standard (first line) treatment. In 2012, 450,000 new cases were observed, leading to death of 170,000 patients [10]. In Europe such multi-drug resistant forms of tuberculosis prevail among the migrants [11].

Treatment of such patients is longer - up to 18-24 months, and is accompanied by severe intoxication [12 Maitre T., et al. 2017,]. The main complication in such patients' treatment is the liver damage with increasing levels of ALT and bilirubin [13,14]. All this requires increasing costs to treat such patients, reaching $€ 140,000$ 220,000 per year $[15,16]$.

Tuberculosis itself is accompanied by long-term and in some cases quite severe intoxication on the background of immunosuppression, which is even more aggravated with activation and long-term course of the inflammatory process. It is no secret that the tuberculosis condition severity depends not only on the underlying disease development, but also on the cumulative effect of many anti-TB drugs. For example, isoniazid, widely used in tuberculosis treatment, overdose or prolonged use of which can cause metabolic acidosis, drowsiness, developing into soporose status and coma, as well as signs of polyneuropathy [17]. Etambutol also 
causes depressive states [18]. Serious toxic complications also occur after rifampicin and pyrazinamide administration [19].

However, the most severe is a toxic liver damage [13,20,21]. The risk of a toxic liver damage is the highest on the background of the present chronic hepatitis and HIV infection [22]. Moreover, the toxic hepatitis risk is the highest in elderly patients [23]. At the same time, the total bilirubin increased level above $5 \mathrm{mg} / \mathrm{dl}$ is a poor forecast [24]. Often there is a hematogenous kidney damage associated with renal failure development requiring hemodialysis [25].

In multi-resistant forms of tuberculosis a more intensive chemotherapy is required with inclusion of 5-6 specific chemotherapy drugs, which is often accompanied by toxic and allergic reactions, manifested in the form of drug hepatitis, urticaria, itching, and skin rashes. Such a forced withdrawal of chemotherapy delays the treatment process and reduces its effectiveness. In addition, tuberculosis is often accompanied by severe intoxication (subfebrile condition, weakness, sweats) associated with the blood rheological properties and microcirculation disorders. All this also makes apheresis therapy indicated [24].

\section{Plasmapheresis}

Performing plasmapheresis courses in toxic hepatitis, which resulted from chemotherapy, appeared to normalize the levels of bilirubin and transaminases, which allowed proceeding with anti-TB therapy $[26,27]$.

On the other hand, a long-term specific inflammatory process itself is accompanied by chronic intoxication associated with development of such severe secondary complications as amyloidosis of the kidneys and other internal organs, which often become the main factor of thanatogenesis. It is also an indication for plasmapheresis.

Tuberculosis is often (up to 20\%) accompanied by bronchial obstruction syndrome, associated with both tuberculin allergy and bronchial hyperreactivity due to intoxication and activation of biologically active substances. In such cases, courses of plasmapheresis with extracorporeal ultraviolet irradiation of the blood enabled to reduce the severity of bronchial obstruction, reduce the daily dose of $\beta_{2}$-agonists, and increase the volume of forced exhalation for 1 second $\left(\mathrm{FEV}_{1}\right)$
The experience of plasmapheresis performed in 1,200 patients with tuberculosis of the respiratory organs and urinary system was reviewed. The indications were endogenous intoxication syndrome, toxic-allergic reactions to anti-TB drugs, liver and kidney dysfunction, hypoxemia and concomitant pathology (bronchial asthma, diabetes mellitus). Positive results were achieved in 93\% of patients, which expanded the possibilities of specific chemotherapy and reduced the incidence of postoperative complications [29]. Plasmapheresis carried out in the preoperative period, in addition to detoxification, improved the state of the lungs surfactant system, which provided better results of surgical treatment in patients with widespread fibrous-cavernous pulmonary tuberculosis [30].

Plasmapheresis was also carried out in 133 patients with various forms of pulmonary tuberculosis (mainly fibrous-cavernous). Following plasmapheresis there was a distinct positive dynamics observed such as decreased signs of intoxication, normalized leukocyte formula (decreased number of stab leukocytes and eosinophils), decreased leukocytosis and ESR [31]. Plasmapheresis efficiency in preoperative conditioning considering marked symptoms of intoxication, signs of toxic hepatitis and chronic DIC-syndrome, as well as in the postoperative period to prevent and treat septic complications [26,30].

In such cases, it was advisable to perform a course of plasmapheresis ( 5 perfusions every 2 days) with subsequent plasmapheresis once a week, and the last plasmapheresis procedure - 2-3 days before the operation. At the same time, in $52 \%$ of patients after 2 months mycobacteria was not detected and in 36\% after 6 months the caverns in the lungs healed. The hospital stay period was significantly reduced up to 2 months [32].

This was especially important in tuberculosis associated with HIV infection, when the combined use of antiretroviral and anti-tuberculosis therapy is accompanied by severe disorders of the bone marrow and liver functions. Relieving such complications by plasmapheresis enables to continue the specific therapy [33]. It should be noted that most patients initially have lymphopenia associated with the underlying disease, and after plasmapheresis there is a normalization of the lymphocytes number, indicating the protective immune status increase. 
Studying coagulation dynamics showed normalization of fibrinogen and fibrinogen " $\mathrm{B}$ ", decreased level of fibrinolytic activity and fibrin stabilizing factor. The prothrombin level increases, which is most likely due to the improvement of protein-synthesizing liver function. It should be assumed that the rheological properties of blood and microcirculation improved helps specific drugs reach the foci of infection more readily, accumulating there to increase the overall effectiveness of the drug therapy [34].

Biochemical blood tests in the process of apheresis therapy indicate normalization of acute-phase proteins (ALT, AST, GGTP, alkaline phosphatase) in $67 \%$ of cases. In other cases, normalization of these findings remains delayed and less complete, which is obviously associated with active toxic or viral hepatitis, and these patients require additional procedures of plasmapheresis with additional administration of intravenous hepatoprotectors (Heptral, Essentiale). The kidney function test shows normalization of the initially impaired blood levels of urea and creatinine [35].

Thus, in such a common and socially significant disease as tuberculosis plasmapheresis can significantly improve the results of its treatment.

\section{Conclusion}

Despite all the achievements of the modern medicine, tuberculosis treatment issue remains unsolved. The main reason is the increasing number of microbacteria strains resistant to most antibiotics and chemotherapy. Such multi-drug resistance forces to administer more drugs simultaneously, which results in severe intoxication, being the leading factor of thanatogenesis. All this makes it necessary to perform apheresis therapy, which, in addition to detoxification also promotes immune correction. Plasmapheresis promotes a faster organ dysfunction recovery and achieving a more stable remission of the disease.

\section{Bibliography}

1. Pezzoli L., et al. "Tuberculosis, Fiji, 2001-2013”. Emerging Infectious Diseases 22 (2016): 547-549.

2. Seybold U. "Tuberculosis among adults in Germany". Deutsche Medizinische Wochenschrift 141.9 (2016): 643-649.

3. Shaweno D., et al. "Heterogeneity of distribution of tuberculosis in Sheka Zone, Ethiopia: Drivers and temporal trends". International Journal of Tuberculosis and Lung Disease 21.1 (2017): 79-85.
4. Salas-Coronas J., et al. "Tuberculosis and immigration". Enfermedades Infecciosas y Microbiología Clínica 34 (2016): 261269.

5. Sánchez MA., et al. "Lethality analysis in patients with tuberculosis diagnosis in a high complexity hospital in Cali, Colombia”. Revista chilena de infectología 35.2 (2018): 133-139.

6. Lun S., et al. "Synthetic lethality reveals mechanisms of Mycobacterum tuberculosis resistance to $\beta$-lactams". MBio 5.5 (2014): e01767-e01714.

7. Galli L., et al. "Pediatric tuberculosis in Italian children: epidemiological and clinical data from the Italian Register of Pediatric Tuberculosis". International Journal of Molecular Sciences 17.6 (2016): e960.

8. Seaworth BJ and Griffith DE. "Therapy of multidrug-resistant and extensively drug-resistant tuberculosis". Microbiology Spectrum 5.2 (2017): 10.1128.

9. Dheda K., et al. "Clinical management of adults and children with multidrug-resistant and extensively drug-resistant tuberculosis". Clinical microbiology and infection 23.3 (2017): 131-140.

10. Seung KJ., et al. "Multidrug-resistant and extensively drug-resistant tuberculosis". Cold Spring Harbor Perspectives in Medicine 5.9 (2015): a017863.

11. Hargreaves S., et al. "Multidrug-resistant tuberculosis and migration in Europe". Clinical microbiology and infection 23.3 (2017): 141-146.

12. Maitre T., et al. "Multidrug and extensively drug-resistant tuberculosis". Médecine et maladies infectieuses 47.1 (2017): 3-10.

13. Yuliwuladari R., et al. "NAT2 variants are associated with druginduced liver injury caused by anti-tuberculosis drugs in Indonesian patients with tuberculosis". Journal of Human Genetics 61.6 (2016): 533-537.

14. Tweed CD., et al. "Liver toxicity associated with tuberculosis chemotherapy in the REMoxTB study". BMC Medicine 16.1 (2018): 46.

15. Diel R., et al. "Cost effectiveness of treating multi-drug resistant tuberculosis by adding Deltybatm to background regimes in Germany". Respiratory Medicine 109 (2015): 632-641. 
16. Musa BM., et al. "Cost-optimization in the treatment of multidrug resistant tuberculosis in Nigeria". Tropical Medicine and International Health21.2 (2016): 176-182.

17. Stettner M., et al. "Isoniazid-induced polyneuropathy in tuberculosis patient - implication for individual risk stratification with genotyping?". Brain and Behavior 5.8 (2015): e00326.

18. Yen YE., et al. "Association of pulmonary tuberculosis and ethambutol with incident depressive disorders: a nationwide, population-based cohort study". Journal of Clinical Psychiatry 76.4 (2015): 505-511.

19. Yee D., et al. "Incidence of serious side effects from first-line antituberculosis drugs among patients treated for active tuberculosis". American Journal of Respiratory and Critical Care Medicine 167.11 (2003): 1472-1477.

20. Gopi M and Seshdri MS. "Biphasic effect of Rifampicin on bilirubin - A case report". Journal of Clinical and Diagnostic Research 10.4 (2016): OD14-OD15.

21. Mushiroda T., et al. "Development of a prediction system for anti-tuberculosis drug-induced liver injury in Japanese patients". Human genome variation 23.3 (2016): 16014.

22. Araújo-Mariz C., et al. "Hepatotoxicity during treatment for tuberculosis in people living with HIV/AIDS". PLoS One 11.6 (2016): e0157725.

23. Hosford JD., et al. "Hepatotoxocity from antituberculous therapy in the elderly: a systematic review". Tuberculosis (Edinb) 95.2 (2015): 112-122.

24. Shigeto E. "Survey of anti-tuberculosis drug-induced severe liver injury in Japan”. Kekkaku (Japan) 82.5 (2007): 467-473.

25. Kanodia KV., et al. "Crescentic glomerulonephritis associated with pulmonary tuberculosis". Journal of Clinical and Diagnostic Research 10.1 (2016): ED01-ED02.

26. Tituhina MV. "Effectiveness of the complex treatment of patients with tuberculosis of different localization in the application of efferent methods of detoxification and nutritional support". Thes. PhD. Dissert. Moscow. (2012): 25.

27. Tityukhina MV., et al. "Use of therapeutic plasma exchange and therapeutic enteral feeding mixtures in patients with pulmonary tuberculosis in the stages of therapeutic and surgical treatment". Efferentnaya Therapia 19.1 (2013): 105-106.
28. Shmelev EI. "Bronchial obstruction syndrome and its correction in patients with pulmonary tuberculosis". Consilium Medicum 6.4 (2004): 254-256.

29. Burov AN and Mikhailov Yu D. "Extracorporeal haemocorrection in tuberculosis". Proc. VII Conf. Moskow society of hemapheresis. Moscow. (1999): 6.

30. Karpina NL., et al. "Experience of small-volume plasmapheresis in correction of homeostasis disorders in patients with progressive destructive pulmonary tuberculosis". Problemy tuberkuleza i bolezner legkikh 11 (2007): 33-37.

31. Zhilin Yu N., et al. "The use of therapeutic plasma exchange in patients with pulmonary tuberculosis". Proc. XIII Conf. Moscow society of hemapheresis. Moscow. (2005): 112-113.

32. Makeeva VG., et al. "Plasmapheresis in complex therapy of patients with acutely progressive pulmonary tuberculosis". Problemy Tuberkuleza 12 (2002): 26-29.

33. Tityukhina MV., et al. "Plasmapheresis in patients with tuberculosis with HIV infection". In Topical issues of hemapheresis, surgical hemocorrection and dialysis. Moscow. (2009): 78.

34. Voinov VA. "Therapeutic apheresis". Constanța: Celebris (2016): 400.

35. Voinov VA., et al. "Membrane plasmapheresis in the treatment of tuberculosis". In: TB. service state and prospects. St. Peterburg: Green Hill. (2005): 185-190.

\section{Volume 2 Issue 8 August 2019 (C) All rights are reserved by Voinov VA., et al.}

